\title{
Minimal Invasive Post Endodontic Monoblock Restorations, II- Sharonlay Crown: Review
}

\section{Abdulsamee $\mathrm{N}^{*}$ \\ Professor and Head of Dental Biomaterials, Deraya University, Egypt}

*Corresponding author: Nagy Abdulsamee, Professor and Head of Dental Biomaterials, Faculty of Dentistry, Deraya University, Egypt, Email: nagyabdulsamee@gmail.com

\section{Editorial}

Volume 6 Issue 3

Received Date: June 25, 2021

Published Date: July 13, 2021

DOI: $10.23880 /$ oajds-16000299

\section{Abstract}

The loss of tooth structure owing to caries, iatrogenic cavity preparation, and dentin drying compromises the structural integrity of root canal treated teeth. Because the quantity of remaining tooth structure and the ability to resist occlusal forces have a direct link, it is critical to offer a post endodontic restoration that allows cuspal covering as soon as possible after completion. The decision to place a full crown or an onlay is based on the remaining tooth structure; an onlay can be inserted if the cuspal width to length ratio is $1: 2$ or above. A full crown must be planned when the ratio is less than 1:2. Cast post and core or a prefabricated post can provide fracture resistance with equivalent outcomes in single-rooted teeth requiring post-endodontic repair. In the case of premolars, however, contrary to the common practice of just providing cuspal covering through onlays, cervical reinforcement would be required to counteract horizontal stresses acting in the cervical region. Only full crown or onlay does not prevent horizontal fracture of tooth that occurs due to shear forces of mastication so many a times radicular support is taken to retain coronal restoration and strengthening of cervical region of tooth. Sharonlay is one of the newer ways being developed by researchers to improve the function of teeth and repair in such circumstances. Sharonlay is a new onlay patented design with I.P. no 1956475 dated 27/04/2010 that has a post extending into the radicular section of the premolar giving the required strengthening in a conservative manner while also protecting it against both vertical and horizontal stresses. It is a potential post-endodontic restoration option for premolars, and it may be indicated in a multirooted tooth primarily for retention, particularly when the clinical crown is too short to support an onlay, crown, or endocrown.

Keywords: Endodontically treated tooth; Post endodontic restoration; Fracture resistance; Onlay; Crown; Sharonlay; Single-rooted premolars; Mandibular second molar

Abbreviations: MID: Minimal Invasive Dentistry, CAD-CAM: Computer Aided Designing-Computer Aided Machining, IOPA: Intra-Oral Peri-Apical.

\section{Introduction}

After root canal treatment, a tooth undergoes numerous changes, including alterations in dentin's physical and chemical characteristics, elasticity, fatigue resistance, morphology, biomechanical behaviour, and loss of proprioception [1]. Since there is a direct relationship between the quantity of surviving tooth structure and the capacity to resist occlusal forces, it is critical to perform a post endodontic restoration that allows cuspal coverage as soon as feasible after root canal treatment is completed [2].

Due to nonaxial loading in single-rooted teeth [3], the role of the post in providing reinforcement has been proven 
[4], regardless of the amount of residual coronal dentin. Even for a post-endodontic restoration, Minimal Invasive Dentistry (MID) requires minimal tooth preparation. The decision to place a full crown or an onlay is based on the remaining tooth structure; an onlay can be inserted if the cuspal width to length ratio is $1: 2$ or above. A full crown must be planned when the ratio is less than 1:2. Cast post and core or a prefabricated post have showed similar long-term effects in single-rooted teeth. However, in the case of premolars, when cuspal covering is the standard, adequate cervical reinforcement is required to oppose the horizontal tension at the cervical area [5]. Sharonlay is a design combining the benefits of onlay with a post that extends into the radicular area of the tooth. The radicular post extension is advised to improve retention because the post in a multi-rooted molar does not improve resistance [6].

When compared to two unit components, single component restorations have a larger surface area for stress absorption, allowing them to withstand higher load before fracturing [7]. The post is often extended 3-5 $\mathrm{mm}$ short of the apex in traditional post and core restorations, however in Sharonlay, the radicular extension can be kept as short as feasible (minimum $7 \mathrm{~mm}$ ) to improve cervical resistance while not weakening the radicular section. When the coronal tooth structure is deteriorated, however, the length of the post can be correspondingly increased to dissipate load resistance and improve retention $[8,9]$.

\section{Indications}

It is recommended for a) single-rooted premolars with short clinical crowns and small vertical dimensions, b) premolars with two roots when one root is parallel to the line of draw of the onlay, c) molars where extra retention is needed, and d) In cases of proximal subgingival margins, where direct restorations are difficult to put, as it is a single unit restoration with only a tooth restoration interface in the subgingival region [10].

\section{Contraindications}

It is not recommended in the following situations: a) severely curved roots, 2) extensive radicular dentin removal to align the post with the Onlay, particularly in the mandibular first premolar where the crown is inclined at $30^{\circ}$ to the root, and 3) severely damaged crowns that cannot support an onlay [11].

\section{Materials Used for fabricating Sharonlay}

It can be manufactured from base metal alloy, cast gold alloy, and zirconia ceramic by computer aided designingcomputer aided machining (CAD-CAM) [12].

\section{Clinical Cases for Sharonlay crown}

Case 1: The post is extended three to five mm short of the apex in traditional post and core restorations, however with Sharonlay, the radicular extension can be kept as short as possible (minimum $7 \mathrm{~mm}$ ) to improve cervical resistance Figure 1a. Because the post is utilised to provide resistance at the neck, retention is not a big concern; nevertheless, if the coronal tooth structure is weakened, the post's length can be increased accordingly. The post's diameter would be determined by the canal's final preparation, which would include modest enlargement with a size no. 3-4 peeso reamer in order to align the post with the overlying onlay and provide enough strength. The onlay is made to protect the tooth's structure and any healthy marginal ridge. The design is created with the cosmetic requirement in mind, as well as the hooding effect essential to avoid crown splitting. Sharonlay is a single-unit restoration made of cast metal, which is the most cost-effective and long-lasting restoration for a single-rooted endodontically treated premolar or molar Figures 1b-h [12].

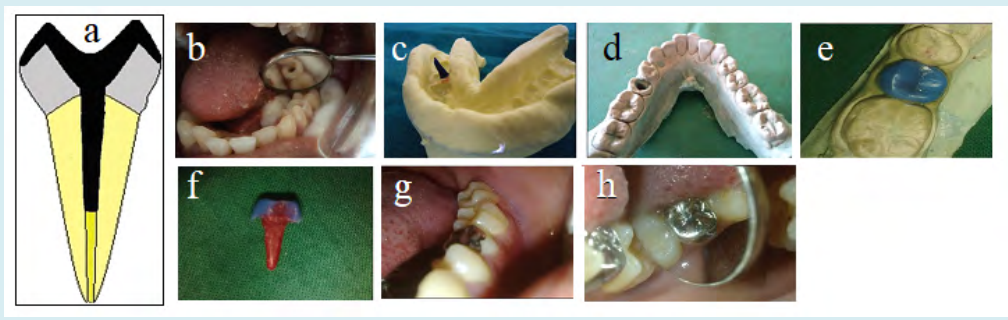

Figure 1: a) Sharonlay design, b) Prepared tooth 35 to receive Sharonlay, c) Impression, d) Master cat, e) Indirect pattern construction for Sharolay, f) Pattern of the Sharonlay restoration, g,h) Finished cemented Sharonlay restoration intra orally [12].

The visibility of metal on the premolars is one of the drawbacks of Sharonlay; however, with the advancement and availability of CAD-CAM technology, the same design may now be manufactured using zirconia. In this case, though, the 
diameter of the post is crucial. A study on the post diameter for zirconia Sharonlay is now underway [12].

Case 2: A 23-year-old female patient with painful tooth 15 presented to the Department of Conservative Dentistry and Endodontics. Because there was no periapical disease and a periapical $\mathrm{x}$-ray revealed pulpal involvement of the tooth Figure $2 \mathrm{a}$, root canal treatment was started and completed in a single visit Figure 2b. Sharonlay was planned as a post endodontic restoration at the next session, taking into account the remaining tooth structure. After completing root canal treatment, it was determined to instal a post in the palatal canal Figure 2c, crown preparation for the Sharonlay Figure $2 \mathrm{e}$, and a rubber base impression was taken because the palatal canal was in line with the draw of the onlay. A base metal cast Sharonlay restoration was produced Figure $2 \mathrm{~d}$ and cemented Figures $2 \mathrm{f}$,g. The radiograph Figure $2 \mathrm{~h}$ [10] shows the restoration of Sharonlay as well as its post with sufficient extension.

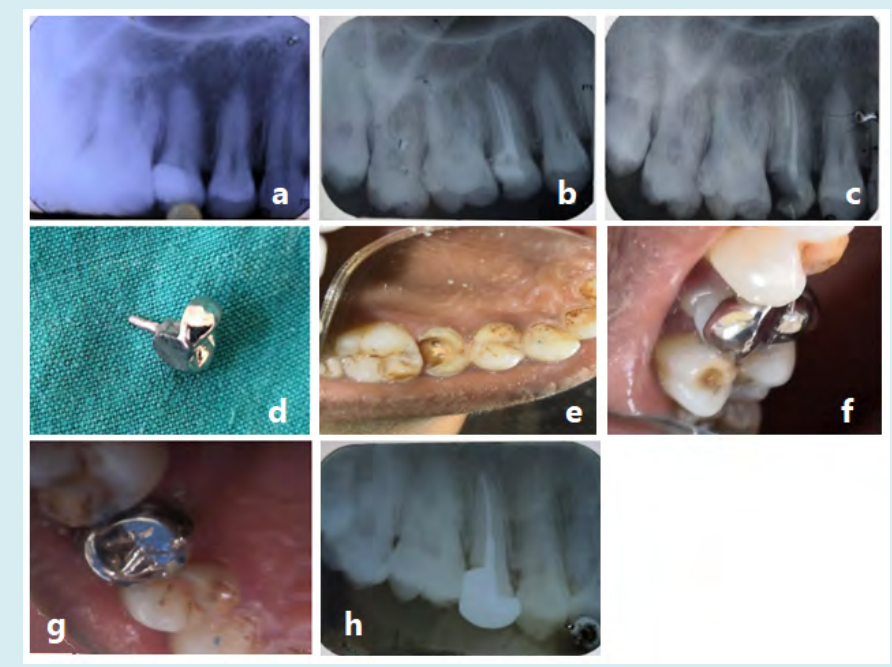

Figure 2: Case 2 Steps of constructing Sharonlay crown for tooth 15 figures a-h.

Case 3: A 28-year-old female patient presented to the Department of Conservative Dentistry and Endodontics with a severe complaint of painful tooth 45 . Figure 3a. On an x-ray, caries that have advanced to the pulp can be seen. The root canal operation was begun and completed in the same visit
Figure 3b. After that, the post space Figure 3c was prepared, the crown Figure 3d was prepared, and an impression was taken Figure 3e. The patient was subsequently brought back for the final cementation of the sharonlay crown for tooth 45 Figures 3f-i [10].

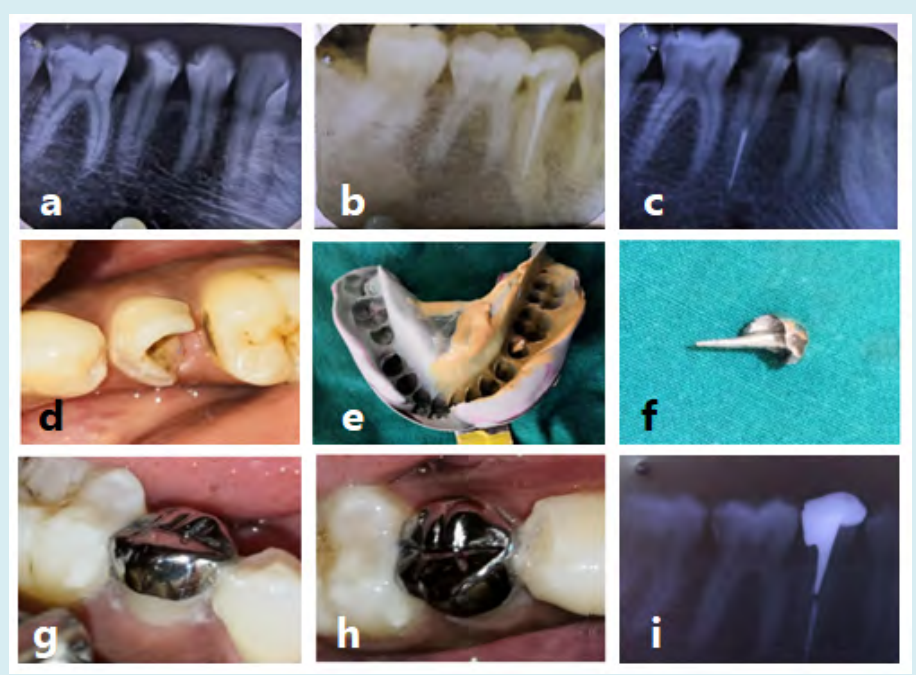

Figure 3: Photos and x-rays for clinical stages of Sharolay crown construction for tooth 45. 
Case 4: First, make root canal treatment. Prepare post hole to a minimum length of $7 \mathrm{~mm}$ from the canal opening, depending on the root length. Depending on the canal configuration and root length, the canal may be expanded more than $7 \mathrm{~mm}$ if more retention is required. If the crown is shorter, a shorter post may be used. To minimise excessive tooth structure removal at the cervical area, the canal is widened with either of the Peeso reamers, Number \# 3, \# 4, \# 5 (equivalent to file sizes 110,130,140), depending on the original canal diameter Figure $4 \mathrm{a}$. The coronal cavity's internal walls are finished with a $5^{\circ}$ taper on each wall. Depending on the material used for the restoration (metal / zirconia) and the amount of residual coronal tooth structure, the buccal and lingual cusps are decreased 1-2 mm Figure 4b. Contact points are preserved, a $0.5 \mathrm{~mm}$ counter bevel is applied to buccal cusps for aesthetic purposes, and a $1 \mathrm{~mm}$ counter bevel is applied to lingual cusps for cosmetic purposes and to achieve the hooding effect Figure $4 \mathrm{~b}$. The impression of the post space is made either directly with inlay wax or indirectly with rubber base material. The first full arch impression is created with heavy body rubber basis material, followed by the impression of the post space with an impression pin and light body rubber base material. A removable die is prepared after the impression, and a wax pattern is created. Casting is carried out using the same material as in a standard casting method. After the casting has been recovered, it is polished and finished as needed, and a try-in is performed on the die before being tried in the patient's mouth. The contour and contact of the patient's mouth are examined before final polishing. After that sharonlay is cemented into the tooth with an adhesive luting cement Figure 4c. Post-operative $\mathrm{x}$-ray film showing well fit Sharonlay restoration of tooth 35 Figure 4d [11].

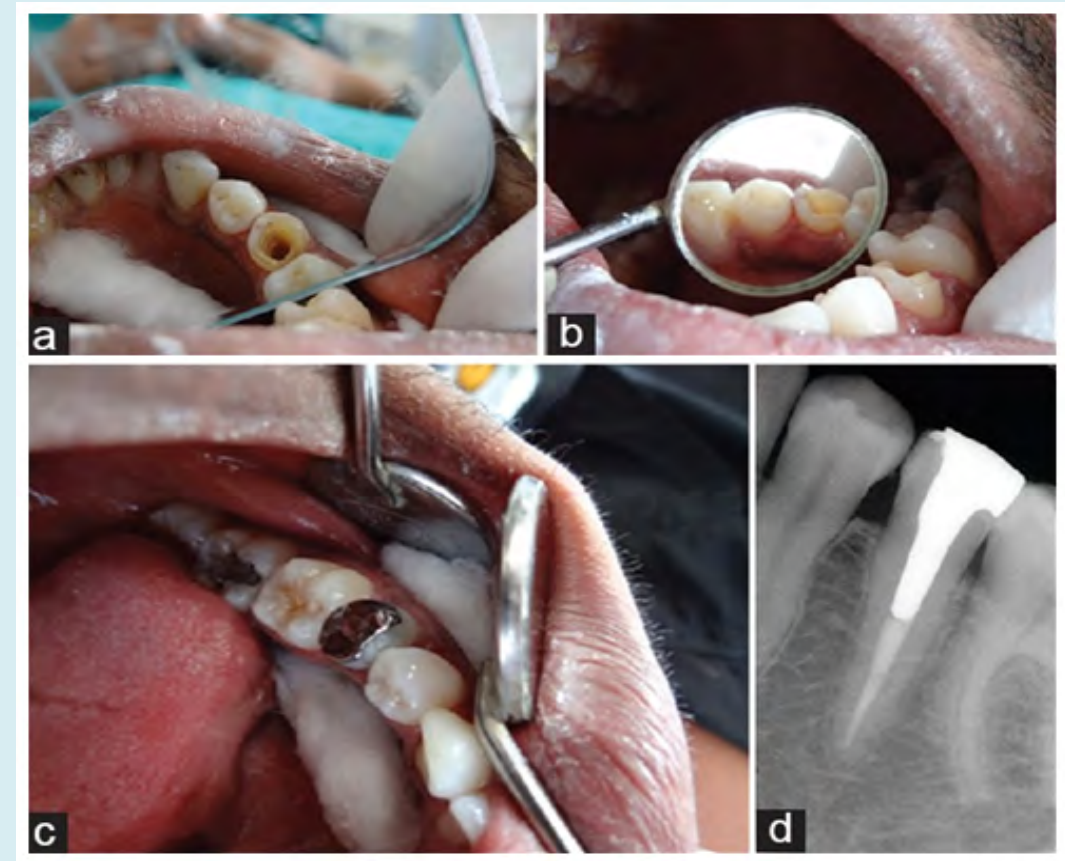

Figure 4: a) Tooth 35 preparation to receive Sharonlay, b) Coronal tooth preparation with buccal and lingual reverse bevel, c) Cemented Sharonlay on the tooth, and d) Postoperative x-ray for the case.

Case 5: The patient complained of tooth 25 being in pain, which was identified as acute irreversible pulpitis and scheduled for standard endodontic treatment. After evaluating the residual tooth structure and the patient's financial situation, Sharonlay was chosen as the post endodontic restoration. There are two canals in the tooth: one palatal and one buccal. Following endodontic treatment, the palatal canal was prepared to receive the post, the buccal was occluded with polycarboxylate cement, and the coronal tooth structure was prepared to receive the onlay. Because the palatal canal coincides with the onlay's line of draw in this design, a single component restoration was possible Figure 5a. The restoration was cast in a chrome-cobalt alloy Figure $5 \mathrm{~b}$ and cemented in the endodontically treated tooth 25 Figure $5 c$. The restoration is seated and contoured on the post cementation radiograph, and the radicular extension of the post is adequate for this patient Figure $5 d$ [13]. 


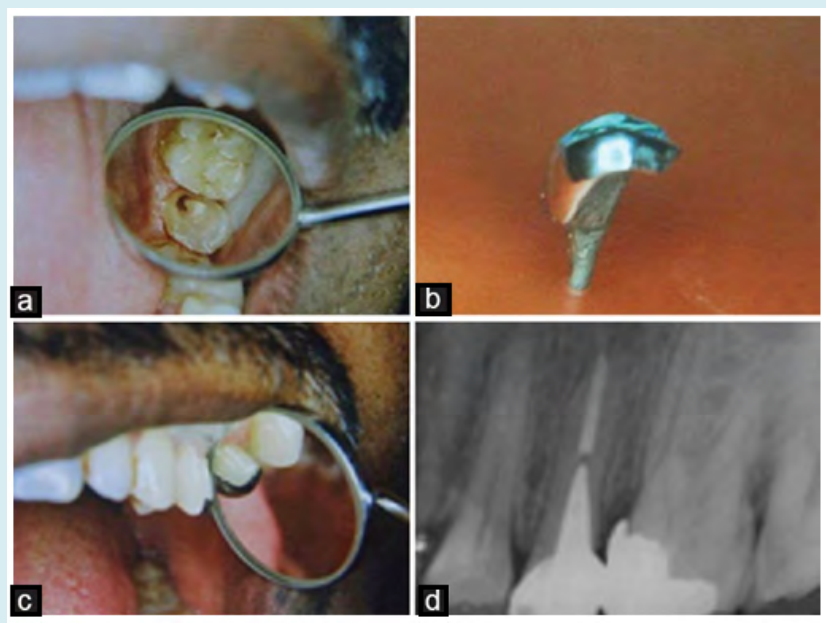

Figure 5: (a) Prepared tooth to receive SHARONLAY on left maxillary second premolar (b) Finished and contoured SHARONLAY (c) SHARONLAY after cementation on left maxillary second premolar (d) Post cementation radiograph on left maxillary second premolars [13].

Case 6 (SHARONLAY in zirconia using CAD-CAM): A 30-year-old male patient presented to my office with a fractured lingual cusp in relation to tooth 25 (maxillary left second premolar), and a 5-year history of root canal therapy. SHARONLAY in zirconia using CAD-CAM (Cerec) was planned with the patient's cosmetic requirements in mind Figures 6a-c. The only drawback of SHARONLAY with ceramic is that it necessitates substantial tooth preparation; if the post is narrow, there is a risk of breakage at the post onlay junction. As a result, the post space preparation must be greater to avoid post fracture, and post lengths more than $7 \mathrm{~mm}$ are difficult to produce using the CAD-CAM method. In this situation, the follow-up is short, as it was sent in June 2012 [13].
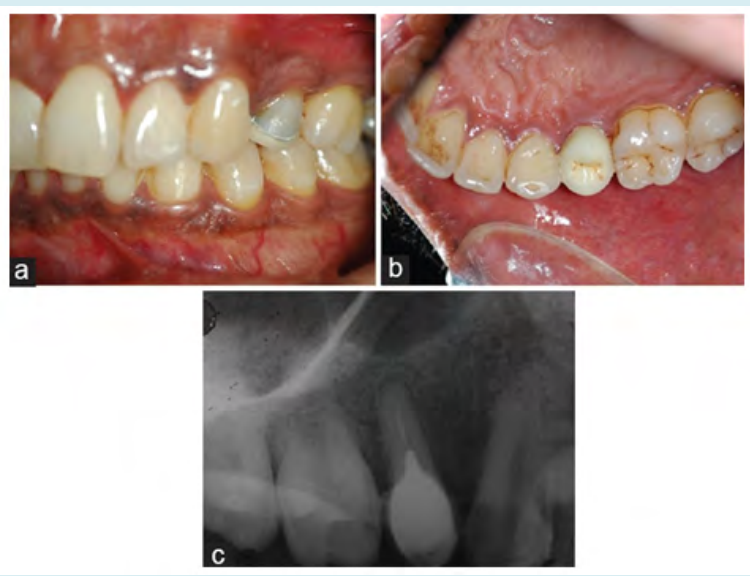

Figure 6: Zirconia Sharonlay restoration on tooth 25 a) buccal view, b) palatal view, and c) Post cementation intraoral periapical (IOPA) on tooth 25 [13].

Fabricating Sharonlay by CAD-CAM technology: CAD-CAM technology can be used to create Sharonlay out of zirconia (Cerec). Because of the potential of fracture at the post-onlay junction if the post is too narrow, it demands extensive tooth preparation. As a result, larger post space preparation is preferred, however CAD-CAM processes fail to prepare post lengths more than $7 \mathrm{~mm}$.

Advantages of CAD CAM: It has an excellent aesthetic, is easy to match shades, can be prepared several times, does not require a traditional imprint manufacturing technique, and does not require laboratory work [11].

Disadvantages of CADCAM:Ithas a number of disadvantages, including the need for prolonged and thorough tooth preparation, the risk of fracture at the post onlay junction if the post is thin, and less conservative preparation [11]. 
Case 7 (Sharonlay crown on molar): Sharonlay may be used in a multirooted tooth for retention purposes only, especially if the clinical crown is too short to support an onlay, crown, or Endocrown. The distal canal, which is in a direct line of draw to the coronal onlay, is used to construct a Sharonlay for the multirooted second molar. It is primarily developed for additional retention as the clinical crown in the case of mandibular second molar is short, especially so when it is the last tooth in the arch and tooth structure not adequate to support an only a crown or an Endocrown. This is the first instance of Sharonlay on a multirooted molar tooth that has been described. A lower second molar was rebuilt with Sharonlay to improve retention Figures $7 \mathrm{a}-\mathrm{c}$ in one example [14].
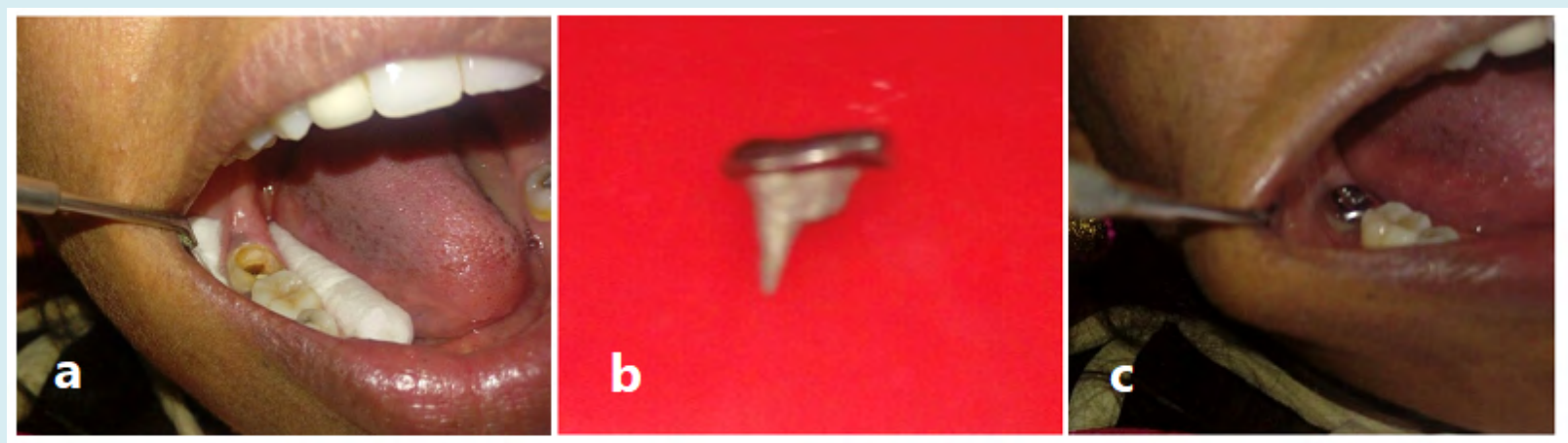

Figure 7: a) Prepared tooth 47 to receive sharonlay, b) Sharonlay casted in Ni-Cr alloy,and c) Sharonlay restoration cemented with GIC luting in the patient mouth after trail [14].

\section{Discussion}

The most common reason for endodontically treated teeth failing is breakage. The amount of healthy dentin left in endodontically treated teeth affects their fracture resistance to horizontal and vertical stresses. The primary goal of endodontic therapy and subsequent coronal restoration is to preserve as much internal dentin as possible. A sufficient amount of healthy dentin tissue is beneficial in preventing fractures caused by vertical and horizontal stresses [15]. The success of a tooth's function following root canal therapy is determined by a number of factors. The degree of healthy dental tissue lost due to disease and iatrogenic causes, as well as the biomechanical alterations caused by root canal therapy, are the essential aspects that lead the physician to restorative treatment planning. According to the literature, the full crown is the gold standard for teeth that have been severely damaged by dental cavities, fractures, or previous conservative-prosthetic preparations [16].

In cases of MO, DO, or MOD preparations, when the tooth is subjected to increased occlusal loading, the posterior teeth become structurally compromised after endodontic treatment. Cuspal deflection causes stress within the tooth, which can result in catastrophic coronal fractures. Based on these findings, it is recommended that after endodontic treatment, all posterior teeth undergo full-coverage restorations [17]. The mandibular first premolar may be the only exception to this guideline. When the lingual cusp of this tooth is underdeveloped, it may not be vulnerable to the wedging forces of opposing cusps when the endodontic access is repaired with an occlusal repair. As a result, in the case of premolars, an onlay is preferable to save more tooth structure. The premolars, on the other hand, are subjected to shear stresses that cause the tooth to fracture at the cervical area. In these circumstances, a post fabrication prevents the fracture by providing cervical support [18].

When enough tooth structure is accessible on both the buccal and lingual sides, as well as for posterior teeth that are subjected to compressive loading, an onlay is the most conservative posterior post-endodontic restoration. When the vertical crown is insufficient for a full crown, an onlay is recommended. Both compressive and tensile forces are applied to the premolars, which are usually single-rooted and positioned anterior to the molars [17]. If solely intra-coronal restorations are done on endodontically treated teeth, cusp fracture is a regular occurrence. Increased cusp deflection during occlusal function is caused by the loss of strategic internal architecture of the tooth, which is particularly prominent in endodontically treated bicuspids [16].

Sharonlay is a design that combines an onlay with a post that extends into the radicular region, resulting in a single component that combines the benefits of both the onlay and the radicular post extension. When compared to two unit components, single component restorations have a larger surface area for stress absorption, allowing them to withstand higher load before fracturing. The post is extended 3-5 $\mathrm{mm}$ short of the apex in traditional post and core restorations, however in sharonlay, the radicular extension can be kept as short as feasible (minimum $7 \mathrm{~mm}$ ) to improve 
resistance at the cervical area. The onlay is made to protect the tooth's structure and any healthy marginal ridge. The design is planned with the aesthetic need in mind, as well as the hooding effect essential to avoid crown splitting $[17,19]$.

A preliminary in vitro investigation compared the fracture resistance of endodontically treated teeth restored utilizing this Sharonlay design to that of untreated teeth. (Group I) has a two-part restoration, i.e., a post with a distinct onlay (separated by $2 \mathrm{~mm}$ ) (Group II). Sharonlay had the highest fracture resistance, with a mean fracture resistance of $514.67 \mathrm{~N}$, which was higher than the maximum voluntary bite force in the premolar region (422 22N in males and 349 $24 \mathrm{~N}$ in females). The fracture lines were found at the cervical level dividing the cusps in both the control group (Group III) and Group II, but they were seen at the apical limit of the post in the Sharonlay group. These data suggest that this unique form provides cervical reinforcement for premolars [20].

\section{Conclusion}

For more than a decade, the Sharonlay design, which is a single component (onlay with post), has been clinically tested. After ten years, a review of these restorations will reveal that this design has a high chance of long-term success [10]. It provides a premolar with greater fracture resistance than (a) a metal onlay with a prefabricated metal post and (b) a metal onlay over an endodontically treated tooth. As a result, Sharonlay could be considered as a post-endodontic restoration option for both upper and lower premolars [7]. The palatal root can be used to try Sharonlay in an upper molar [14]. The visibility of metal on the premolars is one of the drawbacks; however, with the progress and availability of CADCAM technology, the same design may now be created with zirconia [12].

\section{References}

1. Grandini S (2004) Basic and clinical aspects of selection and application of fiber posts. Phd Thesis submitted to university of siena, school of dental medicine, pp: 1-194.

2. Heling I, Gorfil C, Slutzky H, Kopolovic K, Zalkind M, et al. (2002) Endodontic failure caused by inadequate restorative procedures: Review and treatment recommendations. J Prosthet Dent 87(6): 674-678.

3. Heydecke G, Peters MC (2002) The restoration of endodontically treated, single-rooted teeth with cast or direct posts and cores: A systematic review. J Prosthet Dent 87(4): 380-386.

4. Ho MH, Lee SY, Chen HH, Lee MC (1994) Threedimensional finite element analysis of the effects of posts on stress distribution in dentin. J Prosthet Dent
72(4): 367-372.

5. Sapone J, Lorencki SF (1981) An endodonticprosthodontic approach to internal tooth reinforcement. J Prosthet Dent 45(2): 164-174.

6. Salameh Z, Ounsi HF, Aboushelib MN, Sadig W, Ferrari $M$ (2008) Fracture resistance and failure patterns of endodontically treated mandibular molars with and without glass fiber post in combination with a zirconiaceramic crown. J Dent 36(7): 513-519.

7. Sharath Chandra SM, Agrawal N, Sujatha I, Sivaji K (2016) Fracture resistance of endodontically treated single rooted premolars restored with Sharonlay: An in vitro study. J Conserv Dent 19(3): 270-273.

8. Stockton LW (1999) Factors affecting retention of post systems: a literature review. J Prosthet Dent 81(4): 380385.

9. Scotti N, Eruli C, Comba A, Paolino DS, Alovisi M, et al. (2015) Longevity of class 2 direct restorations in rootfilled teeth: A retrospective clinical study. J Dent 43(5): 499-505.

10. Pathak Seema D, Darshana Kale, Bansode PV, Wavdhane MB (2020) SHARONLAY: A new approach for post endodontic restorations: A Case Report. Int J Adv Res, Ideas Innov Technol 6(2): 421-425.

11. Chowdhury D, Priti Desai, Utpal Kumar Das, Antava Mai (2016) Sharonlay-A New Design of Restoration. IDA, WB 32(2): 4-8.

12. Sharath Chandra S, Rahul Singh (2014) Sharonlay - a novel postendodontic restorative design for premolars and single rooted molars: A review. J Oral Res Rev 6(2): 75-79.

13. Sharath Chandra SM (2015) Sharonlay-A new onlay design for endodontically treated premolar. J Conserv Dent 18(2): 172-175.

14. Sharath Chandra SM, Shivani NA, Prabala KV (2019) Sharonlay for a mandibular second molar teeth - A Case Report. Acta Scient Dent Scien 3(1): 93-96.

15. Badr F, Jin Seong W, Perdigão J (2016) Restoring the Endodontically Treated Tooth: Treatment Planning Considerations. Restoration of Root Canal-Treated Teeth pp: 45-66.

16. Barsness BD, Roach SH (2016) Endodontic Considerations for the Restoration of Endodontically Treated Teeth. Restoration of Root Canal-Treated Teeth pp: $1-24$. 
Open Access Journal of Dental Sciences

17. Yoldas O, Akova T, Uysal H (2005) An experimental analysis of stresses in simulated flared root canals subjected to various post-core applications. J Oral Rehabil 32(6): 427-432.

18. Trope M, Maltz DO, Tronstad L (1985) Resistance to fracture of restored endodontically treated teeth. Endod Dent Traumatol 1(3): 108-111.

19. Polesel A (2014) Restoration of the endodontically treated posterior tooth. Giornale Italiano di Endodonzia 28: 2-16.

20. Nishtha A (2014) Unpublished Post-Doctoral Dissertation. Rajiv Gandhi University of Health Sciences; Comparison of fracture resistance of endodontically treated single rooted premolar restored using a cast post and onlay, with a new design-Sharonlay. 